Pacific Journal of Mathematics

ON A REPRESENTATION OF A STRONGLY HARMONIC RING
BY SHEAVES 


\title{
ON A REPRESENTATION OF A STRONGLY HARMONIC RING BY SHEAVES
}

\author{
KWANGIL $\mathrm{KOH}$
}

\begin{abstract}
A ring $R$ is strongly harmonic provided that if $M_{1}, M_{2}$ are a pair of distinct maximal modular ideals of $R$, then there exist ideals $\mathscr{A}$ and $\mathscr{B}$ such that $\mathscr{A} \nsubseteq M_{1}, \mathscr{B} \nsubseteq M_{2}$ and $\mathscr{A} \mathscr{B}=0$. Let $\mathscr{H}(R)$ be the maximal modular ideal space of $R$. If $M \in \mathscr{M}(R)$, let $O(M)=\{r \in R \mid$ for some $y \notin M$, $r x y=0$ for every $x \in R\}$. Define $\mathscr{R}(R)=\bigcup\{R / O(M) \mid M \in \mathscr{M}(R)\}$. If $R$ is a strongly harmonic ring with 1 , then $R$ is isomorphic to the ring of global sections of the sheaf of local rings $\mathscr{R}(R)$ over $\mathscr{C}(R)$. Let $\Gamma(\mathscr{M}(R), \mathscr{R}(R))$ be the ring of global sections of $\mathscr{R}(R)$ over $\mathscr{M}(R)$. For every unitary (right) $R$-module $A$, let $A_{M}=\{a \in A \mid a R x=0$ for some $x \notin M$ ) and let $\tilde{A}=\mathrm{U}\left\{A\left|A_{M}\right| M \in \mathscr{M}(R)\right\}$. Define $\hat{a}(M)=a+A_{M}$ and $\hat{r}(M)=r+O(M)$ for every $a \in A, r \in R$ and $m \in \mathscr{M}(R)$. Then the mapping $\xi_{A}: a \mapsto \hat{a}$ is a semi-linear isomorphism of $A$ onto $\Gamma(\mathscr{K}(R)), \mathscr{R}(R))$-module $\Gamma(\mathscr{M}(R), \widetilde{A})$ in the sense that $\xi_{A}$ is a group isomorphism satisfying $\xi_{A}(a r)=\hat{a} \hat{r}$ for every $a \in A$ and $r \in R$.
\end{abstract}

1. If $R$ is a ring with $1, R$ is called harmonic (or regular) if the maximal modular ideal space, say $\mathscr{L}(R)$, with the hull-kernel topology, is a Hausdorff space (refer [5]). A ring $R$ is strongly harmonic provided that for any pair of distinct maximal modular ideals $M_{1}, M_{2}$ there exist ideals $\mathscr{A}, \mathscr{B}$ in $R$ such that $\mathscr{A} \nsubseteq M_{1}$, $\mathscr{B} \nsubseteq M_{2}$ and $\mathscr{A} \mathscr{B}=0$. For any nonempty subset $S$ of a ring $R$ define $(S)^{\perp}=\{r \in R \mid s r=0$ for every $s \in S\}$ and if $a \in R$ let $a R_{1}$ be the principal right ideal generated by $a$. If $M$ is a prime ideal of a ring $R$ let $O(M)=\left\{r \in R \mid\left(r R_{1}\right)^{\perp} \nsubseteq M\right\}$. An ideal $\mathscr{A}$ of a ring $R$ is called $M$-primary for some maximal modular ideal $M$ of $R$ provided that $M / \mathscr{A}$ is the unique maximal modular ideal of $R / \mathscr{A}$ and if $\mathscr{A}^{\prime}$ is an ideal of $R$ such that $\mathscr{A}^{\prime} \subseteq \mathscr{A}$ and $\mathscr{A}^{\prime} \neq \mathscr{A}$ then $R / \mathscr{A}^{\prime}$ is no longer a local ring (here by a local ring we mean a ring with the unique maximal modular ideal). The principal results in this paper are as follows: Let $R$ be a ring such that if $R / S$ is a local ring for some ideal $S$ of $R$ then $R / S$ has a unit. Then $R$ is strongly harmonic if and only if $O(M)$ is $M$-primary for every maximal modular ideal $M$ of $R$. If $R$ is a strongly harmonic ring with 1 then $R$ is isomorphic to $\Gamma(\mathscr{l}(R), \mathscr{R}(R))$ the ring of global sections of the sheaf of local rings $\mathscr{R}(R)=\bigcup\{R / O(M) \mid M \in \mathscr{C l}(R)\}$ over $\mathscr{C}(R)$ and if $A$ is a unitary right $R$-module then the mapping $\xi_{A}: a \mapsto \hat{a}$ is a semi-linear isomorphism of $A$ onto $\Gamma(\mathscr{C}(R), \mathscr{R}(R))$ - 
module $\Gamma(\mathscr{C}(R), \widetilde{A})$ in the sense that $\xi_{A}$ is a group isomorphism satisfying $\xi_{A}(a r)=\hat{a} \cdot \hat{r}$ for $a \in A, r \in R$ where $\hat{a}(M)=a+A_{M}, \hat{r}(M)=$

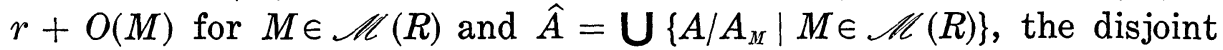
union of the family of right $R$-modules $A / A_{M}$ ) indexed by $\mathscr{L}(R)$, and $A_{M}=\left\{a \in A \mid(a R)^{\perp} \nsubseteq M\right\}$. If $R$ is a ring with 1 such that it contains no nonzero nilpotent elements then $R$ is biregular (see [2: p. 104] for definition) if and only if every prime ideal of $R$ is a maximal ideal. Our results here generalize $S$. Teleman's result that in case $1 \in R$, a strongly semi-simple harmonic ring or a von Neumann algebra can be represented as a ring of global sections of the sheaf of local algebras over its maximal modular ideal space (refer [5], [6] and [7]). The author wishes to express his gratitude to Professors K. H. Hofmann and S. Teleman for their many invaluable suggestions for the preparation of this paper.

2. Let $R$ be a ring and $A$ be a right $R$-module. For each prime ideal $M$ of $R$, define $A_{M}=\left\{a \in A \mid\left(a R_{1}\right)^{\perp} \nsubseteq M\right\}$ where $a R_{1}$ is the submodule of $A$ which is generated by the element $a$ and $\left(a R_{1}\right)^{\perp}=$ $\left\{r \in R \mid a R_{1} r=0\right\}$.

Proposition 2.1. $A_{M}$ is a submodule of $A$.

Proof. Let $a, b \in A_{M}$. Then $(a-b) R_{1} \subseteq a R_{1}+b R_{1}$ and $\left((a-b) R_{1}\right)^{\perp} \supseteqq$ $\left(a R_{1}+b R_{1}\right)^{\perp}=\left(a R_{1}\right)^{\perp} \cap\left(b R_{1}\right)^{\perp} \supseteqq\left(a R_{1}\right)^{\perp}\left(b R_{1}\right)^{\perp}$. Hence if $a-b \notin A_{M}$ then $\left(a R_{1}\right)^{\perp}\left(b R_{1}\right)^{\perp} \subseteq M$ and either $\left(a R_{1}\right)^{\perp} \subseteq M$ or $\left(b R_{1}\right)^{\perp} \subseteq M$ since $M$ is a prime ideal of $R$. Hence either $a \notin A_{M}$ or $b \notin A_{M}$. This is impossible. Thus $a-b \in A_{M}$. Now if $r \in R$ and $a \in A_{M}$ then $a r R_{1} \subseteq a R_{1}$ and $\left(a r R_{1}\right)^{\perp} \supseteqq\left(a R_{1}\right)^{\perp}$. Since $\left(a R_{1}\right)^{\perp} \nsubseteq M,\left(a r R_{1}\right)^{\perp} \nsubseteq M$ and $a r \in A_{M}$.

CoROllary 2.2. If $A$ is $R$, whose module multiplication is given by the ring multiplication, then $A_{M}$ is an ideal of $R$ which is contained in $M$ for any prime ideal $M$ of $R$. In this case, we denote $A_{M}$ by $O(M)$.

Proof. $O(M)$ is already a right ideal of $R$ by 2.2. Let $r \in R$ and $a \in O(M)$. Then $\left(r a R_{1}\right)^{\perp} \supseteqq\left(a R_{1}\right)^{\perp}$. Since $\left(a R_{1}\right)^{\perp} \nsubseteq M,\left(r a R_{1}\right)^{\perp} \nsubseteq M$ and $r a \in O(M)$.

Proposition 2.3. If $A$ is a right $R$-module for some ring $R$ then $A O(M) \cong A_{M}$ for any prime ideal $M$ of $R$.

Proof. Since $A_{M}$ is a submodule of $A$, it suffices to show that if $a \in A$ and $x \in O(M)$ then $a x \in A_{M}$. But this is immediate since $\left(a x R_{1}\right)^{\perp} \supseteqq$ $\left(x R_{1}\right)^{\perp}$ and $\left(x R_{1}\right)^{\perp} \nsubseteq M$. 
Theorem 2.4. Let $R$ be a ring such that if $\mathscr{P}$ is a proper ideal of $R$ then there is a maximal modular ideal $M$ in $R$ such that $\mathscr{P} \cong M$. Let $A$ be a right $R$-module such that if $a R=0$ for some $a \in A$ then $a=0$. Then $\bigcap\left\{A_{M} \mid M\right.$ is a maximal modular ideal of $R\}$ is zero.

Proof. Let $a \in \bigcap\left\{A_{M} \mid M\right.$ is a maximal modular ideal of $\left.R\right\}$ such that $a \neq 0$. Then $\left(a R_{1}\right)^{\perp} \neq R$, for if $\left(a R_{1}\right)^{\perp}=R$ then $a R=0$ and $a=0$. Since $\left(a R_{1}\right)^{\perp} \neq R,\left(a R_{1}\right)^{\perp}$ is a proper ideal of $R$. Hence there is a maximal modular ideal $M$ in $R$ such that $\left(a R_{1}\right)^{\perp} \subseteq M$. This means that $a \notin A_{M}$ and $a \notin \bigcap\left\{A_{M} \mid M\right.$ is a maximal modular ideal of $R\}$. This is a contradiction.

COROLlary 2.5. If $R$ is a ring with 1 and $A$ is a unitary right $R$-module, then $\bigcap\{A O(M) \mid M$ is a maximal ideal of $R\}$ is zero.

Proof. By 2.4, $\cap\left\{A_{M} \mid M\right.$ is a maximal ideal of $\left.R\right\}=0$. Since $A O(M) \subseteq A_{M}$ for any prime ideal of $R$ by 2.3 , the conclusion now follows.

DeFinition 2.6. We say that a ring $R$ is strong harmonic provided that for any pair of distinct maximal modular ideals $M_{1}$, $M_{2}$ there exist ideals $\mathscr{A}, \mathscr{B}$ in $R$ such that $\mathscr{A} \nsubseteq \mathscr{H}_{1}, \mathscr{B} \nsubseteq \mathscr{C}_{2}$ and $\mathscr{A} \mathscr{B}=0$.

Proposition 2.7. If $R$ is strongly harmonic, then $\mathscr{C l}(R)$ is Hausdorff.

Proof. If $M_{1}, M_{2}$ are distinct maximal modular ideals of $R$, then, by definition, there exist ideals $\mathscr{A}$ and $\mathscr{B}$ such that $\mathscr{A} \nsubseteq M_{1}$, $\mathscr{B} \nsubseteq M_{2}$ and $\mathscr{A} \mathscr{B}=0$. Therefore, two open sets $\{M \in \mathscr{C}(R) \mid \mathscr{A} \nsubseteq M\}$ and $\{M \in \mathscr{C}(R) \mid \mathscr{B} \nsubseteq M\}$ are disjoint.

EXAMPLE 2.8. Let $R$ be a strongly semi-simple ring, that is a ring in which the intersection of maximal modular ideals is zero. If the maximal modular ideal space, $\mathscr{C}(R)$ with the hull-kernel topology, is a Hausdorff space, then $R$ is strongly harmonic.

EXAMPLE 2.9. If $R$ is a ring with 1 such that it is strongly harmonic then it is harmonic. However, if $1 \notin R$ then a strongly harmonic ring may not be harmonic. For example, let $R$ be the algebra of sequences $\left(a_{n}\right)_{n \geqq 0}$ of $2 \times 2$-matrices over the field of complex numbers $C$, such that $a_{n} \rightarrow\left(\begin{array}{ll}\lambda & 0 \\ 0 & 0\end{array}\right)$ for $n \rightarrow \infty$ for some $\lambda \in C$. Then 
the intersection of the maximal modular ideals of $R$ is zero and $\mathscr{M}(R)$ is Hausdorff. Hence $R$ is strongly harmonic; however, it is not harmonic.

EXAMPLE 2.10. Let $R$ be a von Neumann algebra. Then for any distinct pair of maximal ideals $M_{1}, M_{2}$ there exist central idempotents $e_{1}, e_{2}$ in $R$ such that $e_{1} \notin M_{1}, e_{2} \notin M_{2}$ and such that $e_{1} \cdot e_{2}=0$. Hence $R$ is strongly harmonic.

ExAmple 2.11. Let $Q$ be the field of rational numbers and let $p_{1}, p_{2}, \cdots, p_{l}$ be a finite number of distinct prime numbers. Let $R=\left\{m / n \in Q \mid n\right.$ is not divisible by any $\left.p_{i}, 1 \leqq i \leqq l\right\}$. Then $\mathscr{M}(R)$ consist of $l$ points and it is a Hausdorff space. However, since $R$ is an integral domain, $R$ is not strongly harmonic if $l>1$.

Definition 2.12. Let $R$ be a ring and $M$ be a maximal modular ideal of $R$. An ideal $\sigma$ in $R$ is said to be $M$-primary, for some maximal modular ideal $M$ of $R$, provided that $O \cong M, R / O$ is a ring with a unique maximal modular ideal $M / O$, and if $P$ is an ideal of $R$ such that $P \cong \mathcal{O}$ and $P \neq \mathcal{O}$, then $R / P$ is not a local ring. Here, by a local ring we mean a ring with a unique maximal modular ideal.

Proposition 2.13. Let $R$ be a ring and $M$ be a maximal modular ideal of $R$. If an M-primary ideal, say $\mathcal{O}$, exists, then it is unique.

Proof. Let $\mathscr{P}$ be a $M$-primary ideal of $R$. If either $\mathscr{P} \subseteq \mathscr{O}$ or $\mathscr{O} \leqq \mathscr{S}$ then, by definition, $\mathscr{P}=\mathscr{O}$. So assume $\mathscr{O} \cap \mathscr{P}$ is properly contained in $\mathscr{O}$ or $\mathscr{P}$. Then the ideal $\mathscr{O} \mathscr{P}$ is properly contained in $\mathscr{O}$ and $R / O \mathscr{P}$ is not a local ring. Hence there is a maximal modular ideal $N$ in $R$ such that $N \neq M$ and $\mathscr{O} \mathscr{P} \subseteq N$. Since $N$ is a prime ideal, this means that either $\mathscr{O} \subseteq N$ or $\mathscr{P} \cong N$. In either case, this means that $\mathscr{O}$ or $\mathscr{P}$ is not $M$-primary. This is a contradiction.

Proposition 2.14. Let $R$ be a ring such that if $R / O$ is a local ring for some ideal $O$ in $R$, then $R / O$ has a unit. If $R / O(M)$ is a local ring for some maximal modular ideal $M$ in $R$, then $O(M)$ is $M$-primary.

Proof. Observe that $O(M) \cong M$. Hence $M / O(M)$ is the unique maximal modular ideal of the local ring $R / O(M)$. Let $\mathscr{P}$ be an ideal of $R$ such that $\mathscr{P} \subseteq O(M), \mathscr{P} \neq O(M)$ and $R / \mathscr{P}$ is a local ring. Let $t \in O(M)$ such that $t \notin \mathscr{P}$. Then $\left(t R_{1}\right)^{\perp} \nsubseteq M$. If $\mathscr{P}+\left(t R_{1}\right)^{\perp} \neq$ 
$R$ then there is a maximal modular ideal $N$ in $R$ such that $\mathscr{P}+\left(t R_{1}\right)^{\perp} \subseteq$ $N$, since $R / \mathscr{P}$ has a unit. Since $\left(t R_{1}\right)^{\perp} \nsubseteq M$, this means that $M \neq N$. This is impossible. Hence $R=\mathscr{P}+\left(t R_{1}\right)^{\perp}$. Let $e+\mathscr{P}$ be the identity of $R / \mathscr{P}$ for some $e \in R$. Then $e=p+s$ for some $p \in \mathscr{P}$ and $s \in\left(t R_{1}\right)^{\perp}$. Hence $t e=t p$ and $t-t e=t-t p \in \mathscr{P}$. This means that $t \in \mathscr{P}$ and this is a contradiction. Thus $O(M)$ must be $M$ primary.

THEOREM 2.15. Let $R$ be a ring such that if $R / O$ is a local ring for some ideal $\mathcal{O}$, then it has a unit. Then $R$ is strongly harmonic if, and only if, $O(M)$ is $M$-primary for every maximal modular ideal $M$ in $R$.

Proof. Assume $R$ is strongly harmonic. By 2.14, it suffices to show that $R / O(M)$ is a local ring for each maximal modular ideal $M$ of $R$. If $R / O(M)$ is not a local ring for some maximal modular ideal $M$, then there is a maximal modular ideal $N$ in $R$ such that $N \neq M$ and $O(M) \subseteq N$. Since $R$ is strongly harmonic, there exist ideals $\mathscr{A}$ and $\mathscr{B}$ such that $\mathscr{A} \nsubseteq N, \mathscr{B} \nsubseteq M$ and $\mathscr{A} \mathscr{B}=0$. This means that $\mathscr{A} \cong O(M)$. Since $O(M) \subseteq N$, $\mathscr{A} \cong N$. This is a contradiction. Conversely, assume $O(M)$ is $M$-primary for each maximal modular ideal $M$ of $R$. Let $M_{1}, M_{2}$ be two distinct maximal modular ideals or $R$. Then $O\left(M_{1}\right) \nsubseteq M_{2}$ and $O\left(M_{2}\right) \nsubseteq M_{1}$. Hence there exist $a \in O\left(M_{1}\right)$ such that $a \notin M_{2}$ and $b \in O\left(M_{2}\right)$ such that $b \notin M_{1}$. Then $(b)$, the ideal generated by $b$, is not contained in $M$. Let $\mathscr{A}=(b)$ and let $\mathscr{B}=\left(b R_{1}\right)^{\perp}$. Then $\mathscr{A} \nsubseteq M_{1}, \mathscr{B} \nsubseteq M_{2}$ and $\mathscr{A} \mathscr{B}=0$.

REMARK 2.16. If $R$ is a strongly semi-simple ring with 1 such that $\mathscr{M}(R)$, the maximal modular ideal space of $R$, is a Hausdorff space, then by [5: Theorem 6.5] and [5: Theorem 6.15], the $M$-primary ideal exists for each maximal modular ideal $M$ in $R$. In this case, the $M$-primary ideal $p(M)$ is given by the set $\{x \in R \mid \overline{\operatorname{supp}(R x R)} \cap\{M\}=$ $\phi\}$, where $\operatorname{supp}(R x R)=\{M \in \mathscr{M}(R) \mid R x R \nsubseteq M\}$ by [5: Theorem 6.14].

3. If $\mathscr{A}$ is an ideal of a ring $R$, let

$$
\begin{aligned}
\operatorname{supp}(\mathscr{A}) & =\{M \in \mathscr{C}(R) \mid \mathscr{A} \nsubseteq M\}, \quad h(A)=\mathscr{C}(R) \backslash \operatorname{supp}(\mathscr{A}), \\
k(F) & =\bigcap\{M \in \mathscr{M}(R) \mid M \in F\} .
\end{aligned}
$$

THEOREM 3.1. Let $R$ be a ring and let

$$
\mathscr{R}(R)=\mathrm{U}\{R / O(M) \mid M \in \mathscr{C l}(R)\},
$$

the disjoint union of a family of rings $\{R / O(M) \mid M \in \mathscr{M}(R)\}$. For 
each $r \in R$ define $\hat{r}$ to be the function from $\mathscr{C}(R)$ into $\mathscr{R}(R)$ such that $\hat{r}(M)=r+O(M)$ for each $M \in \mathscr{C}(R)$. Let $\tau=\{\hat{r}(U) \mid r \in R$ and $U$ is an open set in $\mathscr{C l}(R)\}$. Let $\rho$ be a family of sets consisting of arbitrary unions of the members of $\tau$. Then $(\mathscr{R}(R), \rho)$ is a topological space and each point $\hat{r}(M)$ of $\mathscr{R}(R), r \in R$ and $M \in \mathscr{C}(R)$, is contained in an open set which is homeomorphic to an open set of $\mathscr{l l}(R)$ under the canonical projection: $\hat{r}(M) \mid \rightarrow M$, that is, $\mathscr{R}(R)$ is a sheaf of rings over $\mathscr{C l}(R)$.

Proof. In $\eta \in \hat{r}_{1}(U) \cap \hat{r}_{2}(V)$ for some $r_{1}, r_{2} \in R$ and some open sets $U, V$ in $\mathscr{C}(R)$ then there is $M \in U \cap V$ such that $r_{1}-r_{2} \in O(M)$. Hence $\left(\left(r_{1}-r_{2}\right) R_{1}\right)^{\perp} \nsubseteq M$. Let $\left.W=U \cap V \cap \operatorname{supp}\left(\left(r_{1}-r_{2}\right) R_{1}\right)^{\perp}\right)$. Then $M \in W$ and $\eta \in \widehat{r}_{1}(W) \subseteq \widehat{r}_{1}(U) \cap \widehat{r}_{2}(V)$. Since $W$ is an open set of $\mathscr{L}(R), \hat{r}_{1}(W) \in \tau$ and hence $(\mathscr{R}(R), \rho)$ is a topological space. In view of [1: $2.2 \mathrm{p}$. 151], it suffices to show that if $\hat{r}(M)=0$ for some $r \in R$ and $M \in \mathscr{M}(R)$ then there exists an open set $U$ of $M$ such that $\hat{r}(U)=0$. But this is immediate since if $\widehat{r}(M)=0$ then $r \in O(M)$ and $\left(r R_{1}\right)^{\perp} \nsubseteq M$. Therefore, if we let $U=\operatorname{supp}\left(\left(r R_{1}\right)^{\perp}\right)$ then $\hat{r}(U)=0$ since $r \in \bigcap\{O(M) \mid M \in U\}$.

THEOREM 3.2. Let $R$ be a strongly harmonic ring. If $F$ is a compact subset of $\mathscr{C l}(R)$ and $M_{0} \notin F$ for some $M_{0} \in \mathscr{C l}(R)$ then there exist ideals $\mathscr{A}$ and $\mathscr{B}$ such that $\mathscr{A} \mathscr{B}=O, M_{0} \in \operatorname{supp}(\mathscr{A})$ and $F \subseteq \operatorname{supp}(\mathscr{B})$.

Proof. Since $R$ is strongly harmonic, for any $M \in F$ there exist ideals $\mathscr{A}^{\prime}, \mathscr{B}^{\prime}$ in $R$ such that $M_{0} \in \operatorname{supp}\left(\mathscr{A}^{\prime}\right), M \in \operatorname{supp}\left(\mathscr{B}^{\prime}\right)$ and $\mathscr{A}^{\prime} \mathscr{B}^{\prime}=0$. Since $F$ is compact, there exist a finite number of ideals, say $\mathscr{A}_{1}, \mathscr{A}_{2}, \cdots, \mathscr{A}_{n}, \mathscr{B}_{1}, \mathscr{B}_{2}, \cdots, \mathscr{B}_{n}$ such that

$$
M_{0} \in \bigcap_{i=1}^{n} \operatorname{supp}\left(\mathscr{A}_{i}\right)=\operatorname{supp}\left(\mathscr{A}_{1} \mathscr{A}_{2} \cdots \mathscr{A}_{n}\right)
$$

and $F \subseteq \bigcup_{i=1}^{n} \operatorname{supp}\left(\mathscr{B}_{i}\right)=\operatorname{supp} \sum_{i=1}^{n} \mathscr{B}_{i}$ such that $\mathscr{A}_{i} \cdot \mathscr{B}_{i}=0$ for all $i=1,2, \cdots, n$, and $\left(\mathscr{A}_{1}, \mathscr{A}_{2} \cdots \mathscr{A}_{n}\right)\left(\sum_{v=1}^{n} \mathscr{\mathscr { B }}_{i}\right)=0$.

THEOREM 3.3. Let $R$ be a strongly harmonic ring. If $F$ is a compact subset of $\mathscr{C l}(R)$ then $F=h(\bigcap\{O(M) \mid M \in F\})$.

Proof. Since $\bigcap_{M \in F} O(M) \subseteq k(F), \quad F \subseteq h\left(\bigcap_{M \in F} O(M)\right)$. Suppose there is $M_{0} \in h\left(\bigcap_{M \in F} O(M)\right)$ such that $M_{0} \notin F$. Then by 3.2 there exist ideals $\mathscr{A}, \mathscr{B}$ in $R$ such that $M_{0} \in \operatorname{supp}(\mathscr{A}), F \cong \operatorname{supp}(\mathscr{B})$ and $\mathscr{A} \mathscr{B}=0$. Hence if $M \in F$ then $\mathscr{B} \nsubseteq M$ and $\mathscr{A} \cong O(M)$. Thus $A \subseteq \bigcap_{M \in F} O(M)$. Since $M_{0} \in h\left(\bigcap_{M \in F} O(M)\right)$, this means that $\mathscr{A} \subseteq M_{0}$ and this is a contradiction. 
THEOREM 3.4. Let $R$ be a strongly harmonic ring with 1 and let $\mathscr{R}(R)$ be the sheaf of local rings over $\mathscr{l l}(R)$, which is described in 3.1. If $F_{0}$ is a compact subset of $\mathscr{C l}(R)$ and $\sigma$ is a section from $F_{0}$ into $\mathscr{R}(R)$, then there is $r \in R$ such that $\left.\hat{r}\right|_{F_{0}}=\sigma$.

Proof. If $M_{0} \in F_{0}$ then there exists an open set $U$ in $\mathscr{C}(R)$ which contains $M_{0}$ and $r \in R$ such that if $M \in U \cap F_{0}$ then $\sigma(M)=$ $\hat{r}(M)$. Let $U_{0}=\mathscr{C}(R) \backslash F_{0}$. Since $\mathscr{C}(R)$ is Hausdorff by $2.7, F_{0}$ is a closed set. Hence $U_{0}$ is an open subset of $\mathscr{C}(R)$. There exist a finite number of points $M_{1}, M_{2}, \cdots, M_{n}$ in $F_{0}$, open sets $U_{1}, U_{2}, \cdots, U_{n}$ such that $M_{i} \in U_{i}, i=1,2, \cdots, n$, and $r_{1}, r_{2}, \cdots, r_{n}$ in $R$ such that $\sigma(M)=\widehat{r}_{i}(M)$ for every $M \in U_{i} \cap F_{0}$ for every $i=1,2, \cdots, n$. Furthermore, $F_{0} \subseteq \bigcup_{i=1}^{n} U_{i}$ and $\mathscr{K C}(R)=\bigcup_{i=0}^{n} U_{i}$. Let $F_{i}=\mathscr{K}(R) \backslash U_{i}$ and let $I_{i}=\bigcap_{u \in F} O(M)$ for each $i=0,1,2, \cdots, n$. Since $F_{i}$ is a closed subset of a compact space, it is compact. Hence $F_{i}=h\left(I_{i}\right)$ for each $i=0,1,2, \cdots, n$ by 3.3. Since $\dot{\phi}=\bigcap_{i=0}^{n} F_{i}=\bigcap_{i=0}^{n} h\left(I_{i}\right)=h\left(\sum_{\imath=0}^{n} I_{i}\right)$, $R=\sum_{i=0}^{n} I_{i}$ and $1=\sum_{i=1}^{n} e_{i}$ for some $e_{i} \in I_{i}, i=0,1,2, \cdots, n$. If $M \in F_{i} \cap F_{0}$, then $\hat{r}_{i}(M) \hat{e}_{i}(M)=O(M)=\sigma(M) \hat{e}_{i}(M)$. If $M \in U_{i} \cap F_{0}$, then $\hat{r}_{i}(M) \hat{e}_{i}(M)=\sigma(M) \hat{e}_{i}(M)$. Hence, for every $M \in F_{0}, \hat{r}_{i}(M) \hat{e}_{i}(M)=$ $\sigma(M) \hat{e}_{i}(M)$. Thus if we let $r=e_{0}+\sum_{i=1}^{n} r_{i} e_{i}$, then for every

$$
\begin{aligned}
M \in F_{0} \hat{r}(M) & =\hat{e}_{0}(M)+\sum_{i=1}^{n} \hat{r}_{i}(M) \hat{e}_{i}(M) \\
& =\sigma(M) \hat{e}_{0}(M)+\sum_{i=1}^{n} \sigma(M) \hat{e}_{i}(M) \\
& =\sigma(M)\left(\sum_{i=0}^{n} \hat{e}_{i}(M)\right)=\sigma(M) .
\end{aligned}
$$

COROLLARY 3.5. If $R$ is a strongly harmonic ring with 1 then $R \cong \Gamma(\mathscr{C}(R), \mathscr{R}(R))$.

Proof. By 2.5, $r \mapsto \hat{r}$ is a monomorphism from $R$ into $\Gamma(\mathscr{C}(R)$, $\mathscr{R}(R))$. Since $\mathscr{C}(R)$ is a compact space, by 3.4 if $\sigma \in \Gamma(\mathscr{C}(R)$, $\mathscr{R}(R))$ then there is $r \in R$ such that $\sigma=\hat{r}$. Thus $r \mapsto \hat{r}$ is an isomorphism of $R$ onto $\Gamma(\mathscr{l}(R), \mathscr{R}(R))$.

Definition 3.6. We say that a sheaf $\mathscr{R}$ over the space $X$ is soft provided that if $F$ is a compact subset of $X$ and $\sigma \in \Gamma(F, \mathscr{R})$ then there is $\bar{\sigma} \in \Gamma(X, \mathscr{R})$ such that $\left.\bar{\sigma}\right|_{F}=\sigma$.

THEOREM 3.7. ${ }^{1}$ Let $R$ be a strongly harmonic ring with 1 . Then the sheaf $\mathscr{R}(R)$ of local rings which is constructed in 3.1 is soft. Conversely, if $\mathscr{R}$ is a soft sheaf of local rings over a Hausdorff compact space $/ \mathrm{l}$, then $\Gamma(\mathcal{K}, \mathscr{R})$ is a strongly harmonic ring.

1 The author is indebted to Professor S. Teleman for this theorem. 
Proof. By 3.4, $\mathscr{R}(R)$ is soft if $R$ is a strongly harmonic ring with 1. Suppose now that $\mathscr{R}$ is a soft sheaf of local rings over a Hausdorff compact space $\mathscr{l}$. Let $R=\Gamma(\mathscr{l}, \mathscr{R})$. By Theorem 11 of [6: p. 712], $\mathscr{C}$ is homeomorphic to $\mathscr{C}(R)$. Hence we may take $R=\Gamma(\mathscr{C}(R), \mathscr{R})$. Since $\mathscr{C}$ is Hausdorff, if $M_{1}, M_{2} \in \mathscr{C}(R)$ such that $M_{1} \neq M_{2}$ then there exist open sets $U_{i}, i=1,2$, in $\mathscr{C}(R)$ such that $M_{1} \in U_{1}, M_{2} \in U_{2}$ and $U_{1} \cap U_{2}=\phi$. If $\sigma \in R$, define

$$
|\sigma|=\{M \in \mathscr{M}(R) \mid \sigma(M) \neq 0\} .
$$

Let $A_{i}=\left\{\sigma \in R|| \sigma \mid \leqq U_{i}\right\}, i=1,2$. Clearly, $A_{1}, A_{2}$ are ideals of $R$ and $A_{1} A_{2}=0=A_{2} A_{1}$ since $U_{1} \cap U_{2}=\phi$. There exists compact sets $K_{1}, K_{2}$ such that $M_{i} \in K_{i}$ and $K_{i} \subseteq U_{i}, i=1,2$. Let $F_{i}=\mathscr{C}(R) \backslash U_{i}$. Since $\mathscr{R}$ is soft there exist $\sigma_{i}$ in $\Gamma(\mathscr{C}(R), \mathscr{R})$ such that $\sigma_{i}\left(K_{i}\right)=1$ and $\sigma_{i}\left(F_{i}\right)=0, i=1,2$. Hence $A_{i} \not \subset M_{i}$ for $i=1,2$. Thus $R$ is strongly harmonic.

Remark 3.8. Let $R$ be a ring and $A$ be a right $R$-module. We will associate with $A$ a sheaf if $\mathscr{R}(R)$-modules over $\mathscr{C}(R)$ (refer [4] for definition). For $M \in \mathscr{C}(R)$, denote $\widetilde{A}=\bigcup\left\{A / A_{M} \mid M \in \mathscr{L}(R)\right\}$, the disjoint union of a family of $R$-modules $A / A_{M}$ indexed by $\mathscr{C}(R)$. Let $\pi: \widetilde{A} \mapsto \mathscr{C}(R)$ be given by $\pi^{-i}(M)=A / A_{M}$. For $a \in A$ and $M \in \mathscr{N}(R)$, let $t_{a}(M)$ be the image of $\alpha$, under the natural homomorphism of $A$ onto $A / A_{M}$. Topologize $\widetilde{A}$ by taking all sets $t_{a}(U)$, with $a \in A, U$ is an open set in $\mathscr{C}(R)$, as a basis for the open sets. Then $\widetilde{A}$ becomes a sheaf of $\mathscr{R}(R)$-modules over $\mathscr{C}(R)$. The justification of this statement and proof of this result require only slight modifications of 3.1 .

THEOREM 3.9. Let $R$ be a strongly harmonic ring with 1 and let $A$ be a unitary right $R$-module. Then the mapping $\xi_{A}: a \mapsto t_{a}$ is a semi-linear isomorphism of $A$ onto the $\Gamma(\mathscr{C}(R), \mathscr{R}(R))$-module $\Gamma(\mathscr{C l}(R), \widetilde{A})$ in the sense that $\xi_{A}$ is a group isomorphism satisfying $\xi_{A}(a r)=t_{a} \cdot \hat{\gamma}$ for $a \in A, r \in R$ where $t_{a}(M)=a+A_{s}$ for all $m \in \mathscr{L l}(R)$.

Proof. We omit the proof because it is only a variant of the proof of 3.4. However, it is worth noting that the full strength of 2.4 is needed here to prove that $\xi_{1}$ is an injection.

4. A ring is called biregular if every principal ideal of the ring is generated by a central idempotent. In [2], Dauns and Hofmann proved that if $R$ is a ring with 1 then $R$ is biregular if and only if $R$ is isomorphic to the ring of all global sections of a sheaf of simple rings over a Boolean space. By applying this theorem, we 
will show that if $R$ is a ring with 1 such that it contains no nonzero nilpotent elements then $R$ is biregular if, and only if, every prime ideal of $R$ is a maximal ideal of $R$.

Propostion 4.1. If $R$ is a biregular ring then every prime ideal $M$ of $R$ is a maximal ideal of $R$.

Proof. If $R$ is biregular then so is the ring $R / M$ for any ideal $M$ of $R$. Hence if $M$ is a prime ideal then $R / M$ is a prime biregular ring. Therefore, $R / M$ contains no proper principal ideal for if $R / M$ contains a proper principal ideal, then $R / M$ would have two nonzero ideals whose product is zero. Thus $R / M$ is a simple ring and $M$ is a maximal ideal of $R$.

Proposition 4.2. Let $R$ be a ring and $M$ be a prime ideal of $R$. Define $O_{M}=\{x \in R \mid x y=0$ for some $y \notin M\}$. If $R$ contains no nonzero nilpotent elements then $O_{M}=O(M)$.

Proof. Clearly $O(M) \subseteq O_{M}$. If $x, y$ are elements of $R$ such that $x y=0$ then $y x$ is zero since $y x y x=0$ and $R$ contains no nonzero nilpotent elements. Furthermore, if $r \in R, x r y=0$ since $x r y x r y=0$. Thus $O(M)=O_{M}$.

Proposition 4.3. Let $R$ be a ring without nilpotent elements. If every prime ideal of $R$ is maximal, then $M=O(M)$ for every prime ideal $M$ of $R$.

Proof. If every prime ideal of $R$ is maximal, then every prime ideal is a maximal prime ideal. Hence by [3: 2.4], $M=O_{M}$ for each prime ideal $M$ of $R$. Thus by $4.2 M=O(M)$.

Proposition 4.4. If $R$ is a ring with 1 such that $R$ contains no nonzero nilpotent elements and if every prime ideal of $R$ is maximal, then $\mathscr{C l}(R)$ is a Boolean space.

Proof. This is a direct consequence of [3:2.5].

THEOREM 4.5. Let $R$ be a ring with 1 such that it contains no nonzero nilpotent elements. Then $R$ is biregular if, every prime ideal of $R$ is maximal.

Proof. If $R$ is biregular then by 4.1, every prime ideal is maximal. Conversely, suppose that every prime ideal of $R$ is maximal. Since $R$ is a ring without nilpotent elements, the intersection of 
prime ideals of $R$ is zero. Since $\mathscr{C}(R)$ is a Hausdorff space by 4.4 , if $M_{1}, M_{2}$ are two distinct elements in $\mathscr{C}(R)$, then there exist ideals $\mathscr{A}$ and $\mathscr{B}$ such that $\mathscr{A} \nsubseteq M_{1}, \mathscr{B} \nsubseteq M_{2}$ and $\mathscr{A} \mathscr{B}=0$. Hence $O(M)$ is $M$-primary for every $M \in \mathscr{L}(R)$ by 2.13 and thus $R \cong$ $\Gamma(\mathscr{L}(R), \mathscr{R}(R))$ by 3.5 . Since $\mathscr{C}(R)$ is a Boolean space by 4.4 and $M=O(M)$ by $4.3, R$ is a biregular ring by [2: 2.19, p. 108].

\section{REFERENCES}

1. J. Dauns and K. H. Hofmann, Representation of Rings by Sections, Memoirs, No. 83, 1968, Amer. Math. Soc.

2. - The representation of biregular rings by Sheaves, Math. Zeit., 91 (1966), 103-123.

3. K. Koh, On functional representations of a ring without nilpotent elements, Canad. Math. Bull., (to appear).

4. R. S. Pierce, Modules over Commutative Regular Rings, Memoirs, No. 70, Amer. Math. Soc. (1967).

5. S. Teleman, Analyse harmonique dans les algèbres régulieres, Rev. Roum. Math. Pures, Appl., 12 (1968), 691-750.

6. — La représentation des anneaux reguliers par les faisceaux, Rev. Roum., Math., 14 (1969), 703-717.

7. - Representation of Von Neuman algebras by Sheaves, Acta. Scient. Math. Szeged, (to appear).

Received February 10, 1971.

TUlane UNIVERSITY

AND

North Carolina State University 


\section{PACIFIC JOURNAL OF MATHEMATICS}

\section{EDITORS}

\author{
H. SAMELSON \\ Stanford University \\ Stanford, California 94305

\section{R. HoвBY} \\ University of Washington \\ Seattle, Washington 98105
}

\section{J. DugundJI}

Department of Mathematics University of Southern California Los Angeles, California 90007

\author{
RichaRd ARENS \\ University of California \\ Los Angeles, California 90024
}

\section{ASSOCIATE EDITORS}
E. F. BECKENBACH
B. H. NEUMANN
F. WOLF
K. YoshidA

\section{SUPPORTING INSTITUTIONS}

\author{
UNIVERSITY OF BRITISH COLUMBIA \\ CALIFORNIA INSTITUTE OF TECHNOLOGY \\ UNIVERSITY OF CALIFORNIA \\ MONTANA STATE UNIVERSITY \\ UNIVERSITY OF NEVADA \\ NEW MEXICO STATE UNIVERSITY \\ OREGON STATE UNIVERSITY \\ UNIVERSITY OF OREGON \\ OSAKA UNIVERSITY
}

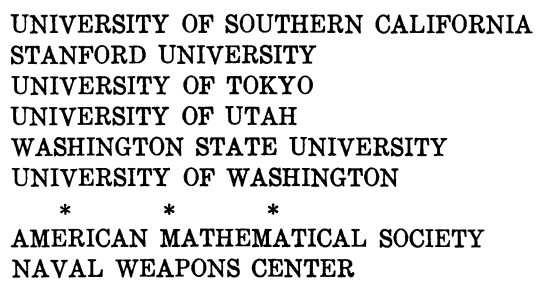

The Supporting Institutions listed above contribute to the cost of publication of this Journal, but they are not owners or publishers and have no responsibility for its content or policies.

Mathematical papers intended for publication in the Pacific Journal of Mathematics should be in typed form or offset-reproduced, (not dittoed), double spaced with large margins. Underline Greek letters in red, German in green, and script in blue. The first paragraph or two must be capable of being used separately as a synopsis of the entire paper. The editorial "we" must not be used in the synopsis, and items of the bibliography should not be cited there unless absolutely necessary, in which case they must be identified by author and Journal, rather than by item number. Manuscripts, in dup icate if possible, may be sent to any one of the four editors. Please classify according to the scheme of Math. Rev. Index to Vol. 39. All other communications to the editors should be addressed to the managing editor, Richard Arens, University of California, Los Angeles, California, 90024.

50 reprints are provided free for each article; additional copies may be obtained at cost in multiples of 50 .

The Pacific Journal of Mathematics is published monthly. Effective with Volume 16 the price per volume (3 numbers) is $\$ 8.00$; single issues, $\$ 3.00$. Special price for current issues to individual faculty members of supporting institutions and to individual members of the American Mathematical Society: $\$ 4.00$ per volume; single issues $\$ 1.50$. Back numbers are available.

Subscriptions, orders for back numbers, and changes of address should be sent to Pacific Journal of Mathematics, 103 Highland Boulevard, Berkeley, California, 94708.

PUBLISHED BY PACIFIC JOURNAL OF MATHEMATICS, A NON-PROFIT CORPORATION

Printed at Kokusai Bunken Insatsusha (International Academic Printing Co., Ltd.), 270, 3-chome Totsuka-cho, Shinjuku-ku, Tokyo 160, Japan. 


\section{Pacific Journal of Mathematics}

\section{Vol. 41, No. 2 December, 1972}

Tom M. (Mike) Apostol, Arithmetical properties of generalized Ramanujan sums .......................................... 281

David Lee Armacost and William Louis Armacost, On p-thetic groups ........ 295

Janet E. Mills, Regular semigroups which are extensions of groups .......... 303

Gregory Frank Bachelis, Homomorphisms of Banach algebras with minimal ideals ................................................ 307

John Allen Beachy, A generalization of injectivity .................. 313

David Geoffrey Cantor, On arithmetic properties of the Taylor series of rational functions. II.........................................

Václáv Chvátal and Frank Harary, Generalized Ramsey theory for graphs. III.

Small off-diagonal numbers .................................. 335

Frank Rimi DeMeyer, Irreducible characters and solvability of finite groups . . . . 347

Robert P. Dickinson, On right zero unions of commutative semigroups........ 355

John Dustin Donald, Non-openness and non-equidimensionality in algebraic

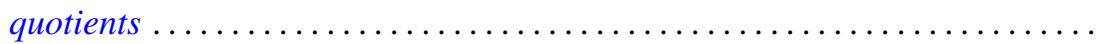

John D. Donaldson and Qazi Ibadur Rahman, Inequalities for polynomials with a prescribed zero ........................................ 375

Robert E. Hall, The translational hull of an $N$-semigroup ................ 379

John P. Holmes, Differentiable power-associative groupoids.............. 391

Steven Kenyon Ingram, Continuous dependence on parameters and boundary data for nonlinear two-point boundary value problems .

Robert Clarke James, Super-reflexive spaces with bases ..........

Gary Douglas Jones, The embedding of homeomorphisms of the plane in

continuous flows...............................

Mary Joel Jordan, Period $H$-semigroups and $t$-semisimple periodic

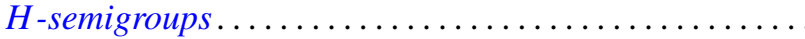

Ronald Allen Knight, Dynamical systems of characteristic 0

Kwangil Koh, On a representation of a strongly harmonic ring by sheaves...

Hui-Hsiung Kuo, Stochastic integrals in abstract Wiener space. ..

Thomas Graham McLaughlin, Supersimple sets and the problem of extending a

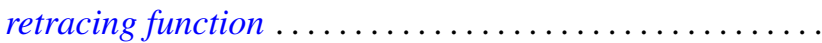

William Nathan, Open mappings on 2-manifolds .

M. J. O'Malley, Isomorphic power series rings

Sean B. O'Reilly, Completely adequate neighborhood systems and metrization

Qazi Ibadur Rahman, On the zeros of a polynomial and its derivative...

Russell Daniel Rupp, Jr., The Weierstrass excess function ..

Hugo Teufel, A note on second order differential inequalities and functional

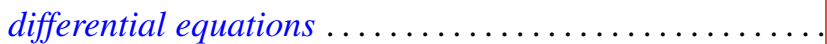

M. J. Wicks, A general solution of binary homogeneous equations over free 